\title{
Gradhiva
}

GRADHIV

Revue d'anthropologie et d'histoire des arts

$23 \mid 2016$

Collections mixtes

\section{Coquilles et médailles. Naturalia et artificialia dans les collections de province autour de la Révolution}

Shells and medals: Naturalia and artificialia in provincial collections around the time of the French Revolution

\section{Pierre-Yves Lacour}

\section{OpenEdition}

Journals

\section{Édition électronique}

URL : http://journals.openedition.org/gradhiva/3130

DOI : 10.4000/gradhiva.3130

ISSN : 1760-849X

\section{Éditeur}

Musée du quai Branly Jacques Chirac

Édition imprimée

Date de publication : 25 mai 2016

Pagination : $50-67$

ISBN : 978-2-35744-093-7

ISSN : 0764-8928

\section{Référence électronique}

Pierre-Yves Lacour, «Coquilles et médailles. Naturalia et artificialia dans les collections de province

autour de la Révolution », Gradhiva [En ligne], 23 | 2016, mis en ligne le 25 mai 2019, consulté le 20 avril 2019. URL : http://journals.openedition.org/gradhiva/3130 ; DOI : 10.4000/gradhiva.3130 


\section{Coquilles et médailles}

Naturalia et artificialia dans les collections de province autour de la Révolution

par Pierre-Yves Lacour

Dans le moment révolutionnaire, tout particulièrement lors des grandes (re)fondations des années 1793-1795, les collections parisiennes tendent à se spécialiser rapidement dans l'un ou l'autre des quatre grands domaines d'objets matériels: la nature, les antiquités, les techniques et les beaux-arts.

Autour de 1800, les collections qui rejettent ce modèle de spécialisation et associent naturalia et artificialia sont démonétisées, à l'image des cabinets provinciaux, qu'ils soient particuliers ou institutionnels. Si, vers 1810 , les collections provinciales mêlent encore des objets naturels et artificiels, ces derniers ne sont plus les mêmes que dans les années 1780, les artefacts exotiques étant tantôt rendus invisibles tantôt ordonnés dans une histoire de l'industrie humaine.

Le couplage coquilles (ou minéraux) et médailles (ou antiquités) est alors la règle. II conviendrait de ne pas lire dans ces mélanges d'objets hétéroclites l'incarnation d'un projet intellectuel de mise en rapport des savoirs mais d'y voir, plus prosaïquement, des rassemblements contingents liés à l'histoire même des collections. 
Les collections mixtes autour de la Révolution mélangent naturalia et artificialia dans le prolongement des cabinets européens du xvII ${ }^{\text {siècle }}$ (Daston et Park 1998; Impey et MacGregor [éd.] 1985; Lugli 1998; Schnapper 1988). Cet assemblage de choses naturelles et artificielles, de spécimens déjà là dans la nature et de produits de la main de l'homme caractérise la culture de la curiosité si prégnante dans l'Europe moderne entre la Renaissance tardive et les premières Lumières, soit à peu près entre 1580 et 1720 . Pourtant, vers 1700, partout en Europe, la curiosité cesse progressivement d'être une valeur connotée positivement (Benedict 2001). Elle n'est certes plus un vice moral comme au Moyen Âge, mais elle est en passe de devenir un défaut de la raison. En quelques années, Bernard Le Bouyer de Fontenelle, René Descartes, Gottfried Wilhelm Leibniz ou Luigi Ferdinando Marsilli dénigrent cet appétit curieux qui mêle des choses de différentes natures. Marsilli, fondateur de l'Institut de Bologne, veut par exemple «absolument montrer le procès de la nature et non pas faire un habit d'Arlequin, ou une mauvaise palette de peintre comme l'ont fait [...] jusqu'à maintenant tous les dilettantes " (Spallanzani 2004: 82). Quelques décennies plus tard, au commencement des Lumières, les cabinets d'histoire naturelle se substituent aux cabinets de curiosités: en 1727, Antoine Joseph Dezallier d'Argenville publie, dans le Mercure de France, une «Lettre sur le choix et l'arrangement d'un cabinet curieux »; en 1742, il consacre un chapitre de sa Conchyliologie à «l'arrangement d'un cabinet d'histoire naturelle» (Pelletier 2012: 72; Lacour 2012: 116). Quelque part entre ces deux dates, les spécialités savantes ont pris la place occupée par la culture de la curiosité, ce dont témoigne encore le cabinet de Joseph Bonnier de La Mosson avec sa petite dizaine de pièces spécialisées vers 1740 . Au cours du xVIII ${ }^{\mathrm{e}}$ siècle, le mouvement de spécialisation des collections s'affirme ainsi pour diverses catégories d'objets, à l'exception des "sauvageries", encore longtemps conservées parmi les choses naturelles.

À la fin du siècle, on voit apparaître dans un catalogue de naturalia l'expression arte facta pour désigner ces «sauvageries", qu'elles soient japonaises, indiennes, chinoises, américaines, tatares ou hottentotes (Gronovius 1778). Dans ces mêmes décennies, le dépérissement des collections curieuses de la bibliothèque de l'abbaye de Saint-Victor et de l'apothicairerie de l'abbaye de Saint-Denis témoigne du long dédain culturel dont font alors l'objet ces artefacts exotiques (Lacour 2014: 53-55). À Paris, leur sort est définitivement scellé lors des grandes confiscations révolutionnaires des collections religieuses et aristocratiques (Sarmant 1994: 226; Daugeron 2009; Lacour 2014: 163-169). Au même moment, comme le souligne Dominique Poulot, «la réorganisation administrative des collections [...] aboutit à une spécialisation des établissements. Elle ne touche cependant que le domaine parisien » (Poulot 1996: 320). On interrogera cette dernière assertion sur le contraste entre Paris et la province en analysant d'abord la mauvaise réputation des collections provinciales, puis les mélanges de spécimens naturels et d'objets de la main de l'homme au sein des cabinets institution-

\section{ci-contre}

fig. 1

Frans II Francken, Kunstund Raritätenkammer, vers 1620-1630, huile sur toile, $86,5 \times 120 \mathrm{~cm}$ Vienne, Kunsthistorisches Museum. nels et, enfin, dans ceux des particuliers.

\section{La mauvaise réputation provinciale}

La période 1793-1795, sous le gouvernement révolutionnaire puis sous le Directoire, voit une véritable refonte des lieux de conservation et l'apparition de nouveaux musées dans l'espace parisien. Le grand «moment 


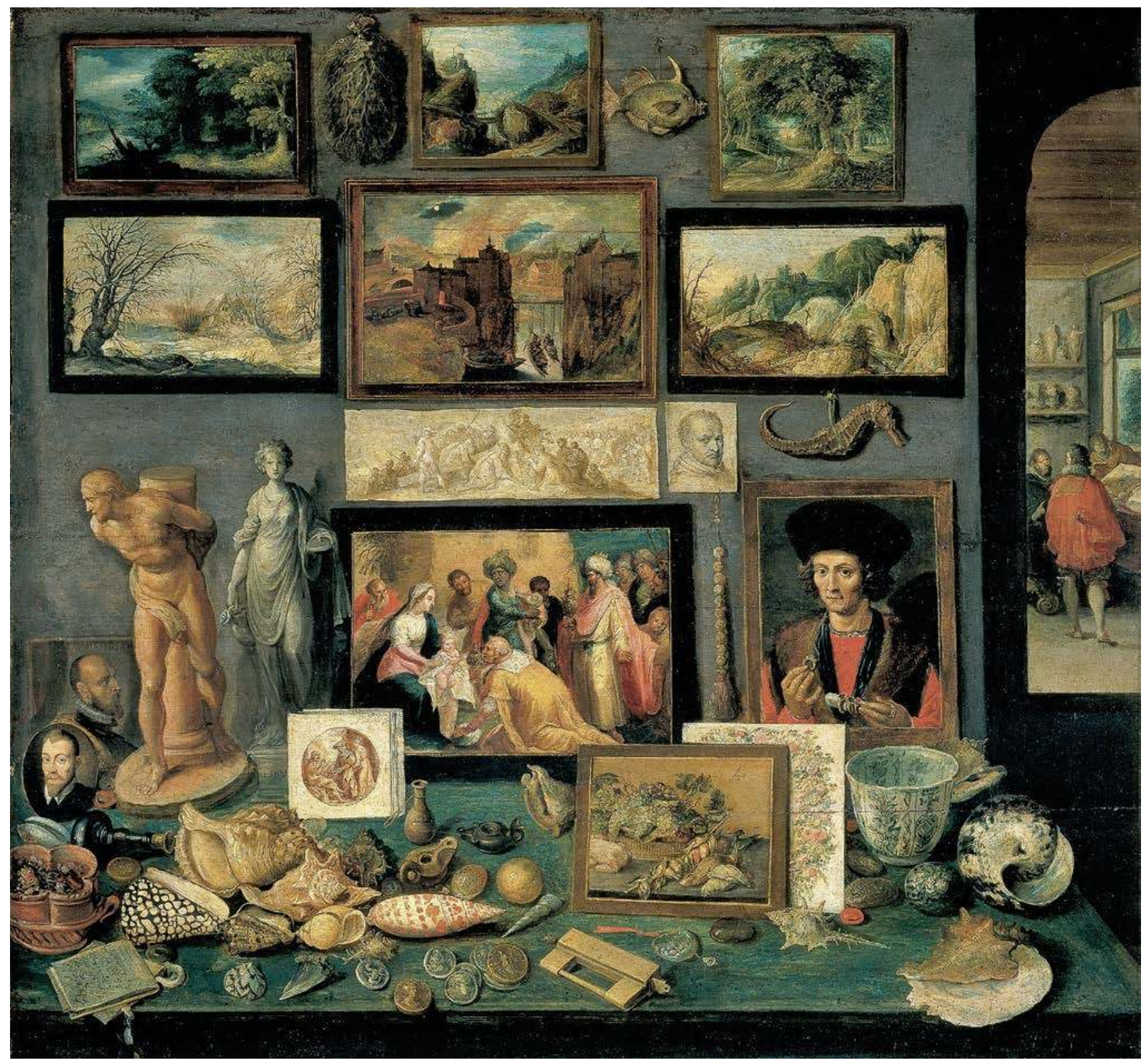


fig. 2

Les quatre pôles muséaux parisiens vers 1800

Infographie de l'auteur. muséologique», comme l'appelle John V. Pickstone (Pickstone 1994), est notamment caractérisé par la création ou la refondation de grandes institutions souvent revêtues du titre de «national » ou de «central »: le Muséum national d'histoire naturelle (1793), le Muséum central des arts (1794), le Conservatoire des arts et métiers (1794) et la Bibliothèque nationale (1795). Chacune de ces institutions doit rassembler des objets selon leur nature et on repère immédiatement quatre grands pôles de spécialisation muséale: les œuvres d'art sont destinées au Muséum central des arts, les livres à la Bibliothèque nationale, les spécimens naturalistes au Muséum national d'histoire naturelle et les machines au Conservatoire des arts et métiers. À l'occasion d'un premier tri, les objets confisqués sont ainsi redistribués entre les différentes collections institutionnelles de la capitale. Tout pourrait dès lors paraître simple: pour chaque chose, une place serait déterminée à l'avance au sein d'une des collections nationales, malgré les incertitudes pour quelques ensembles marginaux d'objets.

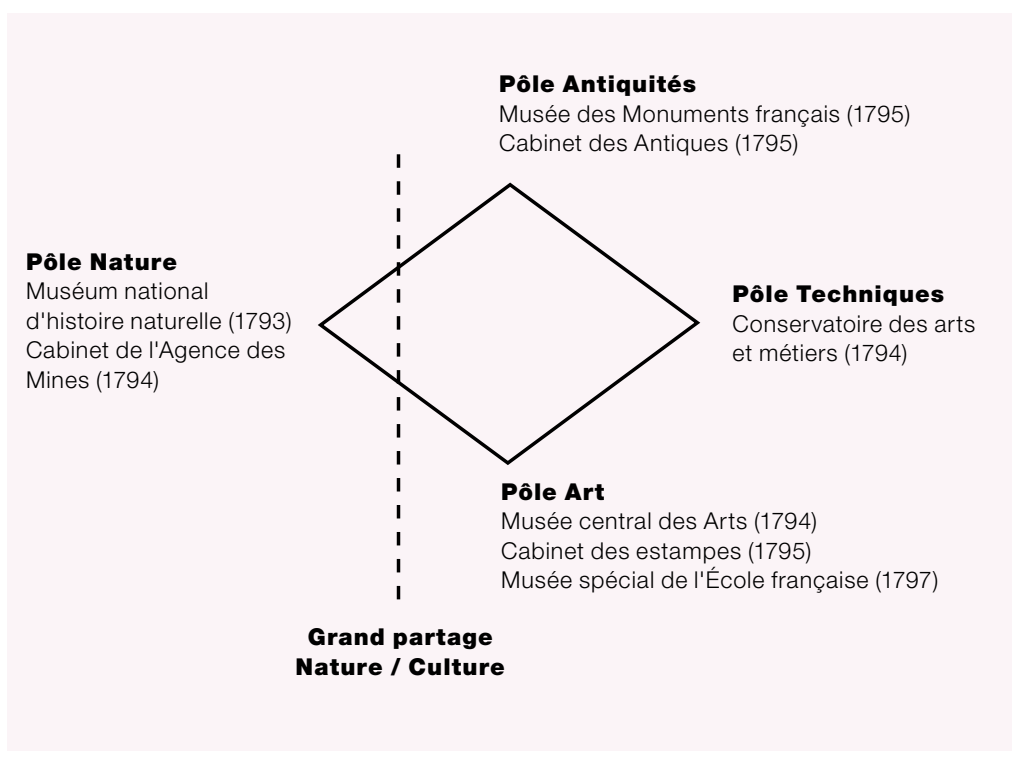

Sous le Directoire, les discours ministériels le récitent à l'envi: il faut en finir avec un ancien régime des collections qui entasse les objets au fur et à mesure qu'ils entrent dans les cabinets et faire cesser les mélanges entre choses naturelles et artificielles. Poulot en a ainsi repéré deux exemples (Poulot 1996: 320-321). Le 8 avril 1796, le ministre Pierre Bénézech recommande à Alexandre Lenoir, conservateur du musée des Monuments français, ainsi qu'«aux conservateurs du Muséum national de ne chercher que le mérite de [ses] collections, et non à entasser des objets qui sont sans caractère dans tel musée et qui en ont un décidé dans tel autre ». Le même écrit aux conservateurs de la Bibliothèque nationale le $1^{\text {er }}$ mai 1796 que son «intention est que désormais chaque Muséum spécial ne contienne que des objets analogues au but de son établissement et qu'ils soient placés dans un ordre méthodique ". Le ministre de l'Intérieur présente ainsi aux conservateurs des grandes collections nationales son projet de rationalisation de la carte des musées de la capitale. II énonce une norme, mais celle-ci n'est pas imposée en province. 
Dans les écoles centrales créées en 1795, la mauvaise réputation des collections mixtes est parfois colportée par des professeurs ou des bibliothécaires, dont celui de Besançon qui écrit en 1796 à propos des collections de médailles:

Monumens de l'histoire dont elles conservent quelques faits ou trais [sic] particuliers sur le bronze, l'airain, le cuivre ou les autres métaux, [les médailles] n'appartiennent pas plus aux arts qui souvent en désapprouveroient le dessin irrégulier qu'à la nature dont elles ne furent jamais une production; cependant je suis informé qu'une partie de ces antiques a été confiée par le département tant au professeur de l'histoire naturelle qu'à celui du dessein! [...]

Si c'est l'intention d'en faire orner le Muséum et le cabinet d'histoire naturelle qui a déterminé ce choix, membre de l'école centrale, je m'opposerai de toute ma force à un mélange hétérogène qui ne pourroit qu'attirer à notre établissement le reproche d'une ignorance crasse ou d'une gaucherie inexcusable. (Troux 1926: 131)

Le bibliothécaire reçoit finalement les médailles avant que le professeur d'histoire s'en mêle, demandant - sans succès - à réunir dans un «cabinet d'archéologie » les médailles et divers «antiques, disséminés dans les cabinets de phisique [sic], d'histoire naturelle et dans la bibliothèque provisoire". On retrouve ici trois des quatre pôles vus précédemment, la nature, les arts et l'histoire, et des tensions pour l'attribution d'objets. Mais plus que le caractère décidé de l'appartenance des médailles aux monuments de l'histoire, ce qui est remarquable ici, c'est la peur de la mauvaise réputation provinciale au regard de la spécialisation des collections de la capitale.

À la suppression des écoles centrales en 1802, ce sentiment de honte devant des collections provinciales jugées trop bigarrées est parfois renversé au nom du vieil idéal encyclopédique. Victor Gueudeville, dans son De l'utilité d'un conservateur près le cabinet d'histoire naturelle et de physique du département du Calvados, publié en 1802, écrit ainsi:

Chaque professeur, me dira-t-on, ne peut-il pas conserver sa partie? Si vous adoptiez ce principe, il n'y a plus de cabinet, et votre responsabilité est compromise. L'historien deviendra le gardien des médailles et des antiques; le physicien réclamera les machines; l'anatomiste s'emparera de toutes les collections ostéologiques, myologiques, et de toutes les injections: professeur d'anatomie comparée, il disputera au naturaliste tout le règne animal, tandis que le chimiste lui enlèvera tout le règne minéral; le médecin demandera les morceaux pathologiques et les objets pharmaceutiques. L'élève découragé se perdra au milieu de ce dédale. (Gueudeville 1802: 8)

L'absence de spécialisation est ici légitimée pour des motifs pédagogiques qui masquent mal la pauvreté de certains cabinets provinciaux comme les ambitions personnelles de Gueudeville, qui pense sans doute à lui-même pour occuper le poste de conservateur qu'il appelle de ses vœux. 


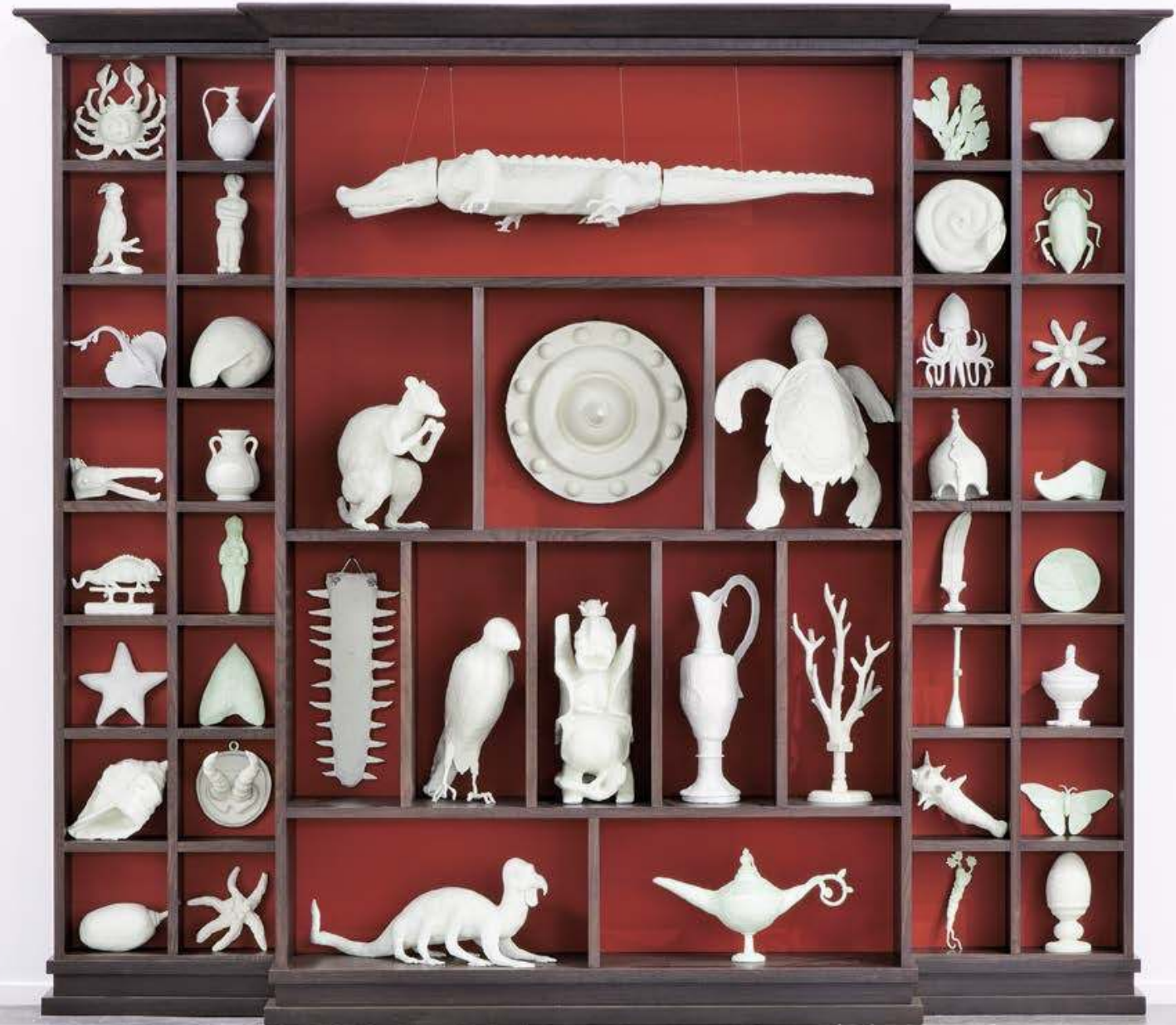




\section{Les collections des institutions}

La distinction entre les cabinets particuliers et les cabinets institutionnels insiste davantage sur la nature de leur propriétaire que sur leur ouverture publique et apparaît mieux taillée pour décrire les cabinets d'Ancien Régime que les notions actuelles et ambiguës de «public» et de «privé» (Lacour 2009). Qu'une collection soit institutionnelle n'est bien sûr pas sans incidence sur son contenu dans la mesure où les institutions s'inscrivent dans des durées plus longues que les vies individuelles, d'où l'aspect parfois désuet de ces collections. En 1780, la dernière édition de la Conchyliologie rédigée par les successeurs de Dezallier d'Argenville ne recense que dix cabinets d'histoire naturelle relevant d'institutions provinciales (Dezallier d'Argenville 1780 [1742]: I, 270-314 et 802-808). Parmi ceux-ci, cinq sont des «collections mixtes» mélangeant artefacts et spécimens.

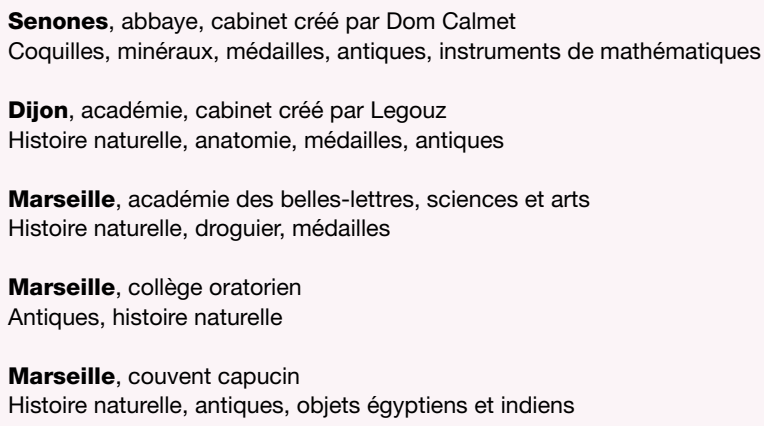

Ces cinq collections provinciales appartiennent à des institutions religieuses, scolaires et académiques. Trois cabinets sont marseillais mais il faut se garder de sur-interpréter cette situation, probablement liée aux informations envoyées aux successeurs de Dezallier d'Argenville par un correspondant local et regroupées dans les additions du volume. Parmi les artefacts, et toujours d'après le même recensement, on repère peu d'exotica à l'exception des objets égyptiens et indiens du couvent des capucins à Marseille. L'association d'objets d'histoire naturelle et de médailles et antiques est, quant à elle, très fréquente. II s'agit ici d'un couplage que l'on retrouvera souvent (sur ce couplage, voir Pomian 1987; Guichard 2012).

Sous le Directoire, à partir de 1797, le Muséum d'histoire naturelle de Paris fait parvenir quantité d'exotica aux cabinets d'histoire naturelle des Écoles centrales de province (Lacour 2014: 166), dont une «racine figurée de la Chine imitant un coq», un «épiderme du bouleau [...] dont on se sert pour écrire", un «petit bateau d'écorce de bouleau des Canadiens", un «modèle de chausses dont se servent les sauvages pour pressurer le manioc", un "casse-tête de sauvage", un «panier des sauvages", et surtout des "flèches de sauvages», souvent empoisonnées, etc. Ces objets sont envoyés par René Louiche Desfontaines, professeur de botanique au Muséum. Dans les catalogues, ils sont intégrés dans la rubrique «Botanique» où ils occupent une place mal définie entre les sous-rubriques «Racines, bois, écorces » et «Supplément». Dans les listes d'envois - comme d'ailleurs dans l'Instruction de Louis-Alexandre de La Rochefoucauld d'Anville tab. 1

Collections institutionnelles mixtes de province en 1780 d'après la Conchyliologie.

\section{ci-contre}

fig. 3

Mark Dion, The Unruly Collection, 2015, cabinet en bois, peinture luminescente et 43 sculptures en papier mâché et plâtre, $220 \times 250$ $x 30 \mathrm{~cm}$. Avec l'aimable autorisation de l'artiste et de la Galerie In Situ www.insituparis.fr (C) Photo Sebastiano Pellion. 
(La Rochefoucauld d'Anville 1790: chap. VII) ou dans les inventaires de saisies -, ces artefacts sont toujours associés aux végétaux, ce qui est une manière de privilégier le matériau qui les constitue sur l'art de leur fabrication, comme l'avait déjà observé Marie-Noëlle Bourguet (Bourguet 1997: 165).

Vers 1800, les nouvelles Écoles centrales disposent souvent de cabinets d'histoire naturelle renfermant des artefacts. À Angoulême, la collection présente des "tissus, carquois et flèches de sauvages" confisqués à des émigrés (Coirault 1940: 122). À Liège, le jury d'instruction fait acheter la collection Preston, dont «une case indienne, avec plusieurs ornements des sauvages" (Macours 1961: 364). Au Mans, la collection renferme six "travaux de sauvages: casse-tête, panier, flèche, bateau, etc. " alors classés dans la section "collection végétale" mais aussi, parmi les "objets précieux", une "améthyste montée en or pour bague épiscopale» (Reinhard 1936: 544). À Nancy, comme dans l'ancien cabinet de curiosités d'Ole Worm, on distingue « une embarcation des Esquimaux avec tout l'attirail de guerre et de pêche " offerte par les héritiers de la veuve Villiez (Gain 1922: 107). À Besançon, dont le cas a déjà été mentionné plus haut, le cabinet contient des flèches, des sabres, un casse-tête, quatre "gibessières [sic] de sauvages ", des bibelots chinois, un lustre orné de coquilles, une statue en marbre de Carrare d'un enfant dormant dans son lit, un «pot de fleur à élytres d'insectes", etc. On trouverait bien d'autres exemples comparables. Ainsi, dans la province révolutionnaire, les œuvres d'art européennes rejoignent les musées artistiques tandis que les «sauvageries" et, dans une moindre mesure, les chinoiseries et les antiquités égyptiennes sont encore destinées aux collections naturalistes. Le grand partage entre collections naturalistes et artistiques en révèle alors un autre, celui qui oppose l'homme naturel à l'homme européen, le Sauvage au Civilisé.

Peu après la suppression des Écoles centrales, les cabinets départementaux dépérissent rapidement, notamment les collections naturalistes souvent dévorées par les insectes (Lacour 2014: 521). Entre 1804 et 1806, Aubin-Louis Millin de Grandmaison entreprend un long périple dans la France méridionale (Hurley 2013: 131-137) dont il publie le récit entre 1807 et 1811 sous le titre de Voyage dans les départemens du Midi (Millin 18071811). Partant de Paris, il prend la route du sud-est, longe la Méditerranée et les Pyrénées, puis remonte vers la capitale en contournant le Massif central. Dans son Voyage..., il présente plus ou moins longuement une petite cinquantaine de cabinets particuliers et institutionnels qu'il a visités. Parmi ceux-ci, il décrit notamment les collections mixtes des municipalités de Marseille, Gap et La Rochelle. Le cabinet d'histoire naturelle de Marseille, formé en 1803 par le préfet Thibaudeau à partir des «débris des cabinets de l'Oratoire et de l'Académie est dans un assez mauvais état: il y a de tout; mais les suites ne sont pas assez complètes pour qu'on puisse dire que ce soit une collection. On y voit le crocodile du Nil, celui d'Amérique; un très grand devin (Boa constrictor), des diodons, des tétrodons, des coraux, des madrépores, comme dans tous les cabinets" mais aussi une "collection de coquilles [qui] présente peu de pièces rares", une autre, "des minéraux [qui] n'offre rien de remarquable ", un droguier "assez complet » ainsi qu'une "collection des médailles [qui] n'est composée que de médailles latines" et "quelques bronzes; quelques idoles et ustensiles antiques; la plupart sont 
des ouvrages égyptiens. On y remarque un assez beau buste de Sérapis, un canope, une Isis chargée de hiéroglyphes, et une momie d'enfant » (ibid. : III, 297-299).

À Gap, «le musée a été fondé en 1801, pour y recueillir tous les objets d'histoire naturelle que présente le département, riche surtout en minéralogie, pour y classer les produits de l'industrie de ses habitans, et les monumens qui peuvent concourir à constater les évènemens de son histoire» (ibid. : IV-I, 171-172). À La Rochelle, «le cabinet d'histoire naturelle est à la maison commune [...]. II contient une riche suite de minéraux, beaucoup de coquilles, des insectes, des madrépores, quelques mammifères, un assez grand nombre d'oiseaux, beaucoup de bocaux qui renferment des amphibies, des reptiles, des poissons, des fœtus et des monstruosités, une pièce de toile d'Otahiti, faite avec les fils du phormium tenax » (ibid.: IV-II, 697). Ces quelques exemples montrent que les collections mixtes des musées de la province postrévolutionnaire ne sont souvent que des rassemblements d'objets hétéroclites où les rares artefacts mêlés à des spécimens d'histoire naturelle ont vocation à éclairer le passé ou l'industrie des hommes.

\section{Les collections des particuliers}

Pour comparer les collections mixtes particulières de part et d'autre de la Révolution, on peut partir de deux publications déjà citées, la Conchyliologie dans sa troisième et dernière édition et le Voyage... de Millin. Ces deux recensements souffrent néanmoins de biais: tropisme parisien dans la Conchyliologie, puisque les cabinets provinciaux ne sont connus que par voyages, correspondances et lectures de catalogues; tropisme antiquaire dans le Voyage..., son auteur abandonnant progressivement, au début du $\mathrm{XIX}^{\mathrm{e}}$ siècle, la botanique pour les antiquités et les inscriptions, motif principal de son périple. En 1780, la Conchyliologie recense cent trente-huit cabinets particuliers en activité pour la province française. Parmi ceux-ci, quatorze associent naturalia et artificialia (Dezallier d'Argenville 1780 [1742] : I, 270314 et $802-808)$. tab. 2

Collections particulières mixtes de province en 1780 d'après la Conchyliologie.
Agey, Marie-Gabrielle de Rochechouart, comtesse Histoire naturelle, "sauvageries", antiques, médailles

Grenoble, Bally de Brochenu, président de la chambre des comptes

Raretés, coquilles, médailles

Avignon, Esprit Calvet, médecin et correspondant de l'Académie des inscriptions

Pétrifications, coquilles, médailles, antiques

Nîmes, Jean-François Séguier, académicien

Pétrifications, coquilles, minéraux, herbier, inscriptions antiques

Dieppe, Féret, apothicaire

Histoire naturelle, médailles, antiques, «sauvageries»

Dieppe, Godebout, échevin et prieur-consul "Sauvageries", poissons, pétrifications, minéraux, coquilles

Vaux-sur-Seulles, Durville, académicien Coquilles, médailles
Angers, Constantin de Monriou, chanoine Coquilles, histoire naturelle, "sauvageries"

Angers, Esnault, avocat

Coquilles, pétrifications, médailles, antiques

Rochefort, Cochon-Dupuy, médecin Histoire naturelle, ouvrages de l'art

Bordeaux, Chazot du Plessis, veuve d'un parlementaire Coquilles, minéraux, histoire naturelle, bijoux antiques

Saint-Dizier, Pierre-Clément Grignon, maître des forges et académicien

Minéraux, antiques

Marseille, Jean-Baptiste Grosson, notaire et académicien Minéraux, coquilles, antiques, médailles

Fécamp, Printemps Duval

Histoire naturelle, curiosités de l'art 
tab. 3

Collections particulières mixtes de province en 1810 d'après le Voyage dans les départements du midi de la France.
Dans ces exemples, il ressort que le couplage le plus commun associe coquilles et/ou minéraux avec médailles et/ou antiques, ainsi dans les cabinets de Bally de Brochenu à Grenoble, d'Esprit Calvet à Avignon, de Durville à Vaux-sur-Seulles, d'Esnault à Angers, de Pierre-Clément Grignon à Saint-Dizier ou de Jean-Baptiste Grosson à Marseille. La plupart de ces collectionneurs sont membres ou correspondants des académies, position qui leur assure de faire partie de l'élite culturelle provinciale (Roche 1978).

On repère également quatre collections contenant des "sauvageries " - des armes et des habillements principalement -, mélangées avec des pièces d'histoire naturelle: celles de Marie-Gabrielle de Rochechouart à Agey, de Constantin de Monriou à Angers, de Féret et de Godebout à Dieppe. La première, dans le parc du château bourguignon d'Agey, est très imposante avec des spécimens des trois règnes de la nature, des instruments de physique et de chimie et des «habillemens \& armes des sauvages, les pierres gravées de composition n'y manquent pas non plus que les antiquités égyptiennes, romaines; une suite de médailles, vases étrusques, lampes, cinéraires, un grand nombre d'estampes, \& de livres curieux" (Dezallier d'Argenville 1780 [1742]: I, 285-286). La dernière, propriété d'un ancien échevin et consul des marchands dieppois, "renferme des arcs, des flèches \& des massues des Caraïbes, une armure complette des Tartares chinois qui consiste en un arc, un carquois rempli de flèches, un sabre avec le bonnet chinois, un bouclier des Malabares fait de cuir peint \& vernissé " (ibid. : I, 305). Ces quatre propriétaires appartiennent à la noblesse titrée, au moyen clergé et au monde des notabilités bourgeoises mais pas à l'univers des élites savantes, à la différence des propriétaires des cabinets d'histoire naturelle et d'antiquités, le plus souvent académiciens.

Entre 1807 et 1811, Millin décrit dans son Voyage... neuf collections particulières réputées «mixtes» (Millin 1807-1811).

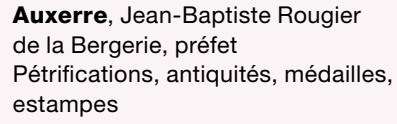

Auxerre, Jean-Baptiste Rougier de la Bergerie, préfet

Pétrifications, antiquités, médailles, estampes

Dijon, Antoine, ingénieur

Minéraux, médailles, curiosités, dessins

Autun, Legouz, chanoine

Histoire naturelle, médailles

Lyon, «ll Romano », commerçant

Minéraux, coquilles, médailles, antiques

Viviers, Flaugergues, juge de paix

Minéraux, antiques
Avignon, Esprit Calvet, médecin Minéraux, antiquités, médailles

Apt, Sigoïer

Coquilles, minéraux, médailles

Marseille, veuve Gauthier Minéraux, coquilles

Nîmes, Jean-François Séguier, académicien décédé Minéraux, pétrifications, antiques
Ces collections bigarrées sont le plus souvent modestes, au moins pour certaines: le cabinet de Jean-Baptiste Rougier de la Bergerie à Auxerre ne possède que "quelques morceaux d'antiquité", celui d'Antoine à Dijon est une "petite collection ", celui du chanoine Legouz à Autun est formé des débris d'un ancien cabinet plus important, soit «quelques médailles très communes, quelques échantillons d'histoire naturelle et quelques livres ", 
et celui d'“II Romano" n'est qu'un «amas confus et poudreux » de minéraux, de coquilles, de médailles et d'antiques, souvent des faux.

Les cabinets de Calvet et de Jean-François Séguier - même si le premier est un vieillard et si le second n'est plus depuis un quart de siècle - se distinguent dans cette dizaine de collections autant par leur richesse incomparable que par leur mobilisation dans les pratiques savantes de leur propriétaire (Cavalier 2011 ; Pugnière 2016). Selon Millin, Calvet est:

à la fois savant naturaliste et savant antiquaire; il possède une riche collection de productions du règne minéral et d'antiquités, un grand nombre de vases pour la vie civile et pour les sacrifices, des ustensiles de différentes espèces, des figurines, un précieux médaillier, et beaucoup d'inscriptions. Son âge avancé et ses infirmités sont souvent un obstacle au désir qu'on auroit de voir sa collection, et le rendent peu accessible. Je ne puis que me louer de ses bontés et de son gracieux accueil; mais je n'osais lui demander la permission de prendre une notice détaillée de son précieux cabinet, ou plutôt je vis bien qu'elle me seroit refusée. (Millin 1807-1811: II, 168)

Ainsi, au début du $x x^{\mathrm{e}}$ siècle, on ne repère plus guère que le couplage coquilles et/ou minéraux avec médailles et/ou antiques. Les artefacts exotiques ont, semble-t-il, complètement disparu.

Entre 1780 et 1810 , soit le temps d'une bonne génération de part et d'autre de la Révolution, la disparition des exotica est également repérable dans la comparaison entre deux cabinets naturalistes nantais: celui de Kérambart à la veille de la Révolution et celui de Buron et Dubuisson qui, peu après 1800, forme le premier fonds du Muséum d'histoire naturelle de Nantes. La collection Kérambart appartient à une femme, probablement veuve d'un officier de marine. En janvier 1798, l'inventaire qui en est dressé propose une estimation de chacune des pièces ${ }^{\mathbf{1}}$. Les spécimens d'histoire naturelle - individus zoologiques et échantillons minéralogiques - représentent $83 \%$ de la valeur totale du cabinet, le reste rassemblant des «objets d'art», des tableaux, des gravures et des figurines, mais aussi des antiquités, des chinoiseries et des "sauvageries", et quelques pièces curieuses. Tout cela fait de la collection Kérambart un cabinet naturaliste encore marqué par la curiosité et caractéristique d'une certaine culture nobiliaire de la fin du XVIII ${ }^{e}$ siècle. À la charnière des deux siècles, elle est absorbée par le cabinet Buron comme le prouve le passage d'une collection à l'autre de quelques pièces exceptionnelles. En 1801, la collection Buron, dirigée par François-René-André Dubuisson, fait à son tour l'objet d'un inventaire et d'une prisée des pièces ${ }^{2}$. Le cabinet, à la différence de celui de la veuve dont il est principalement issu, ne renferme que très peu d'«ouvrages de l'art», moins de $2 \%$ de sa valeur totale. De manière étonnante, d'après le catalogue imprimé, ces quarante artificialia sont aux trois quarts des «sauvageries ", les chinoiseries et les œuvres d'art européennes ayant alors presque complètement disparu (Dubuisson 1799: 243-244). Surtout, le sens de ces artefacts a changé avec la redéfinition de leurs usages: ces «quelques productions de l'industrie [...] peuvent donner une idée des arts chez les peuples sauvages, et forment, pour ainsi dire,
1. Inventaire du cabinet de Kérambart par Huet, Peccot, Dubuisson, Buron, Kérambart. Nantes, du 16 au 24 nivôse an VI, archives municipales de Nantes, 2R 568.

2. Inventaire de la collection Buron par Athenas, Dubuisson, Richard, Noüel. Nantes, du 27 brumaire au 17 frimaire an X, archives municipales de Nantes, 2R 567. 


\section{ci-contre}

fig. 4

Vue du cabinet de Joseph Bonnier de la Mosson (1702-1744), reconstitué à la Bibliothèque centrale du Museum d'histoire naturelle de Paris. Photo (C) Muséum national d'histoire naturelle, Dist. RMN-Grand Palais / image du MNHN Bibliothèque centrale. les élémens de l'histoire industrielle» (ibid. : III). Les exotica ont donc ici une position imprécise entre ce que nous avions défini comme le pôle de la nature et celui des techniques.

\section{Conclusion}

Au sein des cabinets de la province postrévolutionnaire, l'ancien couplage entre naturalia et artificialia est encore à l'œuvre, à la différence de ce que l'on observe dans les musées parisiens précocement spécialisés au cours de la Révolution. La continuité avec le monde des cabinets de la fin de l'Ancien Régime semblerait alors patente. II faut néanmoins apporter quelques nuances qui modifient profondément l'interprétation globale. D'une part, les artificialia ne sont plus qu'exceptionnellement des "sauvageries " et sont généralement des antiquités, tout au moins pour les collections particulières. L'assemblage entre coquilles et/ou minéraux et médailles et/ ou antiques est le type même de la collection «mixte » provinciale vers 1800 . D'autre part, le mélange entre spécimens naturels et artefacts ne traduit pas un projet intellectuel qui associerait savoirs antiquaires et savoirs naturalistes et dont on ne retrouverait qu'exceptionnellement la trace ailleurs. Dans le meilleur des cas, il éclaire plutôt les manières de faire partagées entre deux démarches savantes qui reposent sur l'observation, la comparaison et la description d'objets matériels. Mais, le plus souvent, ce mélange résulte à l'évidence de la réunion contingente et souvent malheureuse de débris de collections disparues.

Dans les collections provinciales du début du $x x^{e}$ siècle, les exotica sont parfois encore présents sans pour autant être mentionnés dans les descriptions qui en sont faites par les savants et les autorités publiques. Ainsi, le cabinet d'histoire naturelle de Nancy renferme comme on l'a vu d'assez nombreux artefacts, mais il n'en est fait aucune mention dans la description qu'en propose le conservateur pour le Magasin encyclopédique (par comparaison Gain 1922: 107; Magasin encyclopédique, $9^{e}$ année, vol. V, 1804: 126-127). Cela prouve, mieux encore que les transferts entre collections, le déclassement de ce type d'objet à l'aube du nouveau siècle. Vers 1800 , en province et dans la capitale, les artefacts exotiques semblent passer par trois des quatre pôles que nous avions identifiés. Ils intègrent généralement les collections d'histoire naturelle en province, les musées antiquaires dans la capitale et, exceptionnellement, les cabinets à visée industrielle dans la mesure où ils peuvent "contribuer à la perfection de nos arts \& de nos manufactures", comme l'indique en 1790 La Rochefoucauld d'Anville (La Rochefoucauld d'Anville 1790: chap. V), et dix ans plus tard Dubuisson. La nature, les antiquités et les techniques sont ainsi les trois places possibles pour les exotica en ce début de siècle mais, à aucun moment, ceux-ci ne sont envisagés comme des œuvres d'art et destinés aux musées des beaux-arts.

Plus tard, au milieu du xIx ${ }^{e}$ siècle, pour paraître moderne, on propose encore de démembrer les collections mixtes. Le médecin Eugène-Pierre Derostaing-Derivas écrit ainsi à propos des collections du Muséum d'histoire naturelle de Nantes: 


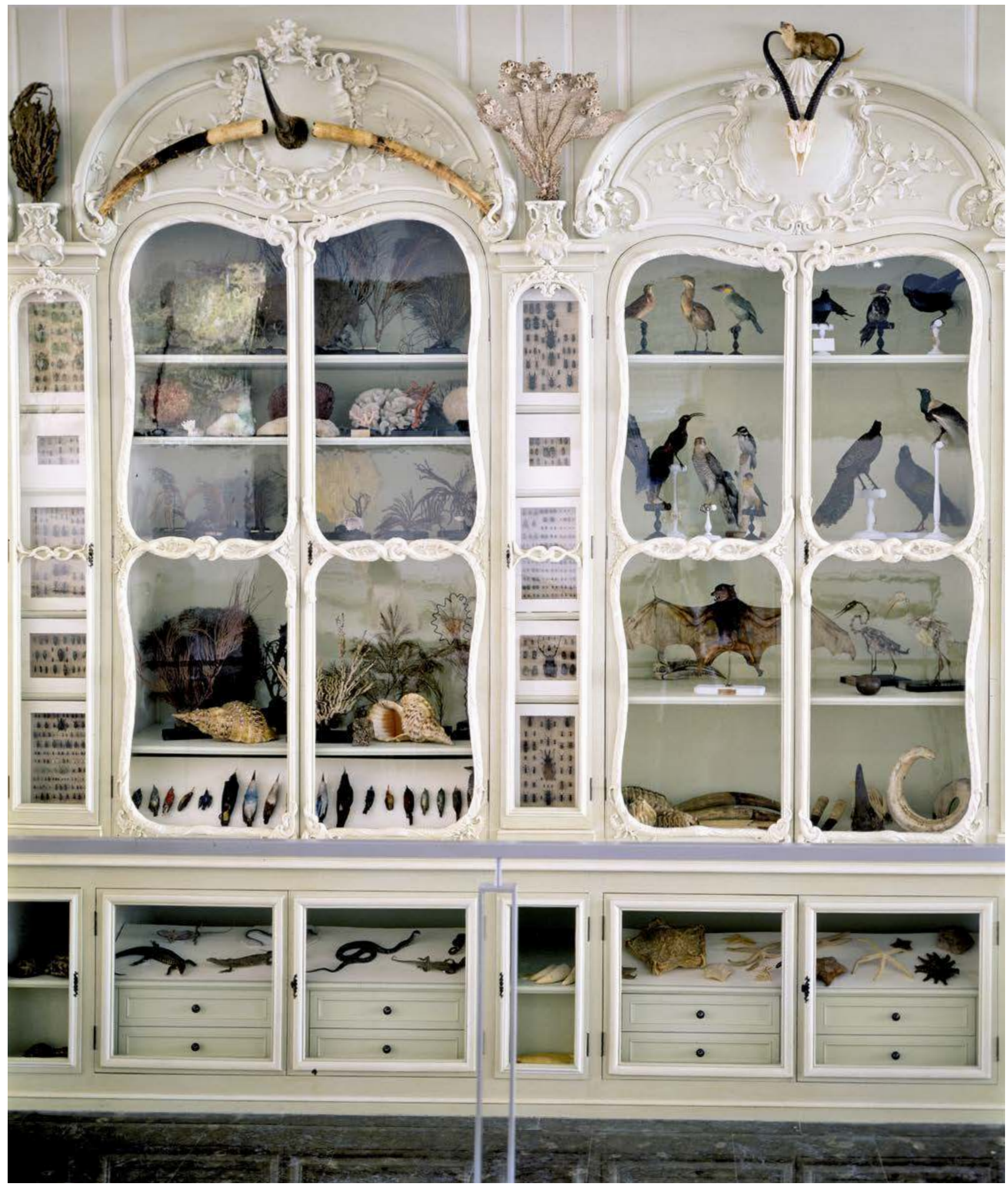




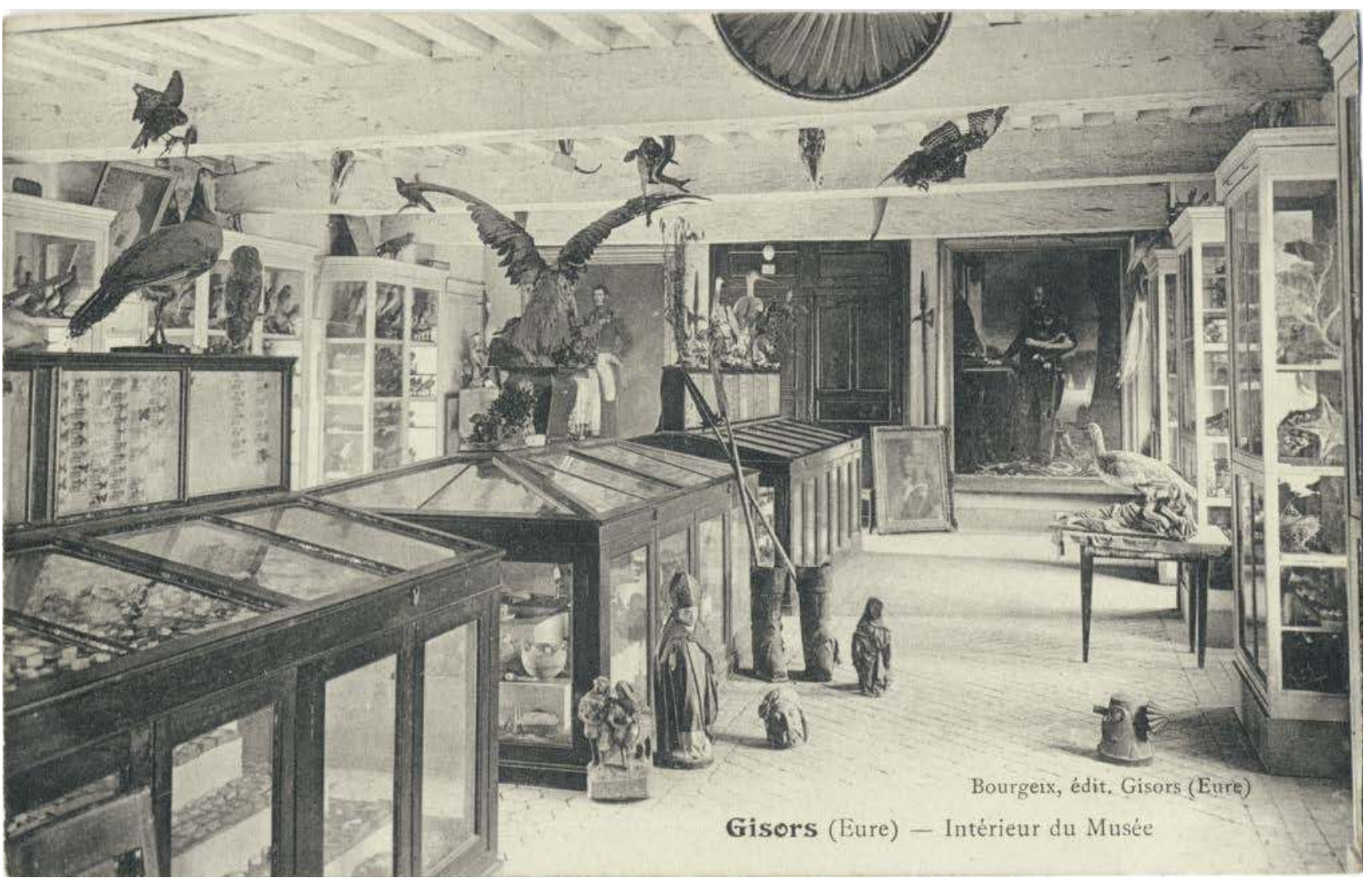

fig. 5

Gisors (Eure) - Intérieur

du musée, Bougeix editeur.

Gisors. Carte postale,

vers 1910, coll. part. 
Chaos, à la vérité, qui n'existe pas seulement dans l'établissement de notre ville; nous le retrouvons pour ainsi dire partout en province. On y prend un cabinet d'histoire naturelle pour un entrepôt général. Ainsi, dans notre musée, on trouve des momies entourées de leurs bandelettes, des amulettes égyptiennes, péruviennes, des armures, etc. Pourquoi ne pas placer ces antiquités dans un cabinet de la bibliothèque publique, et les tombeaux qui en ornent l'avenue dans le musée archéologique que va organiser l'Association bretonne? Ces pièces pathologiques figureraient beaucoup mieux à l'école de médecine. Enfin tous ces bocaux renfermant des graines, toutes ces plantes plus ou moins mal préparées, pourquoi ces produits de la végétation ne sont-ils pas dans la salle du Jardin des plantes, avec la collection qui existe déjà ou devrait y exister? (Derostaing-Derivas 1847: 142)

Ainsi, «l'habit d'Arlequin » que moquait Marsilli un siècle et demi plus tôt est encore dénoncé à Nantes en plein cœur du XIXe siècle, signe de la persistance de la mauvaise réputation des collections provinciales. 
(dir.), 1740, un abrégé du monde.

Savoirs et collections autour

de Dezallier d'Argenville. Lyon,

Fage Éditions : 60-74.

\section{Pickstone, John V.}

1994 «Museological science? The place of the analytical/comparative in Ninenteenth-Century science, technology and medecine", History of Science 32: 111-138.

\section{Pomian, Krzysztof}

1987 «Médailles/coquilles = érudition/philosophie", in Collectionneurs, amateurs et curieux. Paris, Venise $x V I^{e}-x V I I^{e}$ siècle. Paris, Gallimard ("Bibliothèque des histoires"): 143-162.

\section{Poulot, Dominique}

1996 "Surveiller et s'instruire». La Révolution française et

l'intelligence de l'héritage historique. Oxford, Voltaire Foundation.

\section{Pugnière, François}

2016 «De l'Instrumentarium au Muséum. Le cabinet

de Jean-François Séguier

(1703-1784) », Liame [en ligne], 26

\section{Reinhard, Marcel}

1936 Le Département de la Sarthe sous le régime directorial.

Saint-Brieuc, Les Presses bretonnes.

page 50 et ci-contre Mark Dion, The Unruly Collection, 2015 , cabinet en bois, peinture luminescente et 43 sculptures en papier mâché et plâtre $220 \times 250 \times 30 \mathrm{~cm}$ Avec l'aimable autorisation de l'artiste et de la Galerie In Situ www.insituparis.fr (C) Photo Sebastiano Pellion.
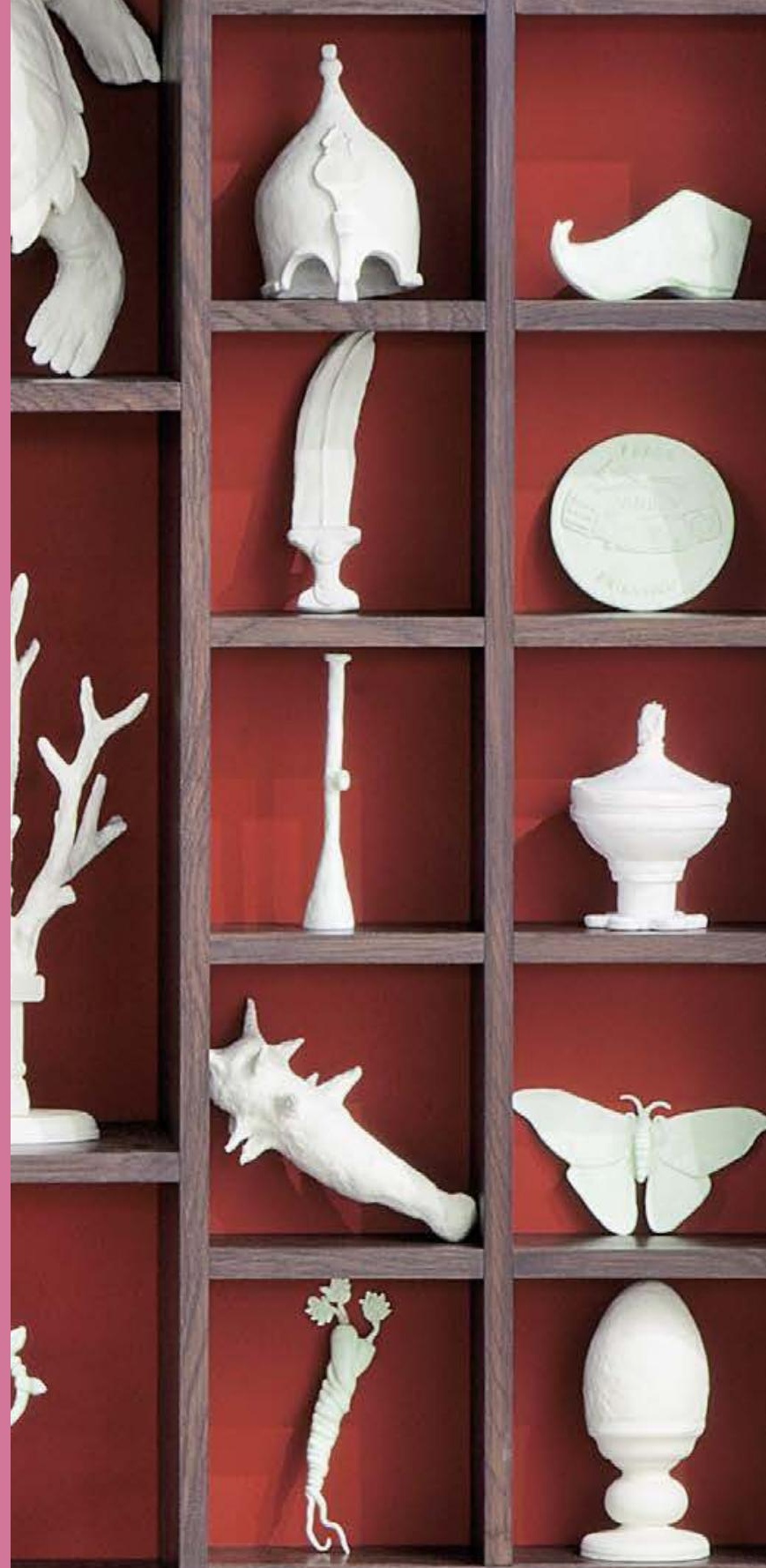